International Journal of Algebra, Vol. 1, 2007, no. 8, 375 - 380

\title{
Prime Submodules in Multiplication Modules
}

\author{
Atul Gaur, Alok Kumar Maloo and Anand Parkash \\ Department of Mathematics and Statistics \\ Indian Institute of Technology, Kanpur 208016, India \\ atulgaur@iitk.ac.in, akmaloo@iitk.ac.in, anandpar@iitk.ac.in
}

\begin{abstract}
An example of a ring $R$ and a multiplication $R$-module $M$ is given such that every prime submodule of $M$ is cyclic but $M$ is not. Some sufficient conditions for a multiplication module to be cyclic have also been given.
\end{abstract}

Mathematics Subject Classification: 13C13, 13C99

Keywords: Multiplication Modules, Prime Submodules

\section{Introduction}

Let $R$ be a commutative ring with unity and $M$ be an $R$-module. The notion of prime submodules in $M$ is an analogue of the notion of prime ideals in $R$. Indeed, prime submodules of $R$ are precisely its prime ideals. Therefore, it is of interest to find out whether some results on prime ideals also hold for prime submodules.

The prime avoidance theorem has been proved by C. P. Lu, see [6, Theorem 2.3]. He proves the result under a condition and also gives an example which shows that without the condition the result is not true, in general. In view of this, we are encouraged to consider the following two statements:

(a) If all prime submodules of $M$ are finitely generated then $M$ is Noetherian.

(b) If all prime submodules of $M$ are cyclic then so are all submodules of $M$.

Note that these statements are true in $R$ (see, Ex 2.22 and Ex 2.23 in [4]). Unfortunately, neither of these statements holds if $M$ is an arbitrary $R$-module. A countre-example for (a) is given in this note (see, Example 3.1) and for a countre-example for (b), take $R$ to be a field and $M=R^{2}$. 
However, in [3], statement (a) has been proved for a special class of modules, known as multiplication modules. Therefore, in this note we investigate if statement (b) also holds for multiplication modules. In fact, we show by an example that statement (b) is not true even for multiplication modules (see, Example 3.3).

We also determine some sufficient conditions for (b) to be true for a multiplication module.

\section{Preliminaries}

Throughout this article, rings are assumed to be commutative with unity and modules are assumed to be unitary.

Let $R$ be a ring. We recall the following definition:

Definition 2.1 A proper submodule $N$ of an $R$-module $M$ is said to be a prime submodule of $M$ if $a x \in N$ for $a \in R$ and $x \in M$ then either $a M \subseteq N$ or $x \in N$.

We remark that if $N$ is a prime submodule of $M$ then $P=(N: M)$ is necessarily a prime ideal of $R$ and therefore $N$ is sometimes referred as a $P$-prime submodule of $M$.

We now recall another definition:

Definition 2.2 An $R$-module $M$ is said to be a multiplication $R$-module if for each submodule $N$ of $M$ there exists an ideal $I$ of $R$ such that $N=I M$.

An ideal $I$ of $R$ is said to be a multiplication ideal of $R$ if $I$ is a multiplication $R$-module.

Remark 2.3 Let $M$ be a multiplication $R$-module. Then all quotients of $M$ are multiplication $R$-modules. Furthermore, if $S \subset R$ is a multiplicative set then $S^{-1} M$ is a multiplication $S^{-1} R$-module.

Submodules of $M$ need not be multiplication $R$-modules.

If $N$ is a submodule of $M$ then $N=(N: M) M$.

We now recall some results on multiplication modules from [5]: Let $M$ be a multiplication module over a ring $R$.

(1) [Theorem 2.5] If $M$ is nontrivial then $M$ admits maximal submodules. If $N$ is a maximal submodule of $M$ then $N=\mathfrak{m} M$ for some maximal ideal $\mathfrak{m}$ of $R$. Furthermore every proper submodule of $M$ is contained in a maximal submodule.

(2) [Theorem 3.1] If $M$ is faithful then $M$ is finitely generated if and only if $I M \neq M$ for all proper ideals $I$ of $R$. 
(3) [Corollary 3.3] If $R$ is Noetherian then $M$ is Noetherian.

(4) [Corollary 2.11 and Theorem 2.5] Let $P$ be a prime ideal of $R$ containing the annihilator of $M$. If $P M \neq M$, then $P M$ is a $P$-prime submodule of $M$ and if $P$ is maximal then so is $P M$.

(5) [Lemma 4.1] If $M$ is faithful then $M$ is torsion free.

\section{Results}

We recall (see, Ex. 2.22 of [4]) the following theorem of I. S. Cohen: Let $R$ be a ring such that every prime ideal of $R$ is finitely generated. Then $R$ is Noetherian.

However, if every prime submodule of an $R$-module $M$ is finitely generated then $M$ is not necessarily Noetherian as can be seen from the example given below.

Example 3.1 Consider $\mathbb{Q}$, the field of rational numbers, as a $\mathbb{Z}$-module. Let $N$ be a prime submodule of $\mathbb{Q}$ and let $P=[N: \mathbb{Q}]$. Then $P$ is a prime ideal of $\mathbb{Z}$ and $P \mathbb{Q} \subseteq N$. Since $N \neq \mathbb{Q}$, we must have $P=(0)$. We now claim that $N=(0)$. Suppose $N \neq(0)$. Then there exist nonzero integers $a$ and $b$ such that $a b^{-1} \in N$. As $N$ is (0)-prime, we see that $b^{-1} \in N$ and therefore $1 \in N$, that $i s, \mathbb{Z} \subseteq N$. As $N \neq \mathbb{Q}$, there exist nonzero integers $\alpha$ and $\beta$ such that $\alpha \beta^{-1} \notin N$. However, $\beta \alpha \beta^{-1}=\alpha \in N$. This is a contradiction as $\beta \neq 0$ and $N$ is (0)-prime.

Therefore the only prime submodule of $\mathbb{Q}$ is the submodule $(0)$ and $\mathbb{Q}$ is not finitely generated over $\mathbb{Z}$.

Interestingly, the result does hold for multiplication modules and has been proved by M. Behboodi and H. Koohy (see, [3, Corollary 3]). We provide here an alternative proof, which appears more natural.

Theorem 3.2 Let $R$ be a ring and let $M$ be a multiplication $R$-module such that every prime submodule of $M$ is finitely generated. Then $M$ is Noetherian.

Proof. We may assume that $M \neq\{0\}$. Then, by [5, Theorem 2.5], $M$ admits a maximal submodule, say $L$. As $L$ is maximal, there exists $x \in M$ such that $M=L+R x$. As every maximal submodule is also prime, $L$ is finitely generated and therefore so is $M$.

Suppose, if possible, $M$ is not Noetherian. Let

$$
\mathfrak{F}=\{N \mid N \text { is not a finitely generated submodule of } M\} .
$$


Then $\mathfrak{F} \neq \emptyset$ and $M \notin \mathfrak{F}$. We order $\mathfrak{F}$ by inclusion. Now, by Zorn's lemma, $\mathfrak{F}$ has a maximal element, say $N=I M$, where $I=(N: M)$. We show that $I$ is prime. Clearly $I \neq R$. Let $a, b \in R$ such that $a b \in I$. Suppose $a \notin I$ and $b \notin I$. Then $a M \nsubseteq N$ and $b M \nsubseteq N$. In particular, $N+a M$ is finitely generated.

Therefore $N+a M=\sum_{i=1}^{n} R x_{i}$. Hence $x_{i}=t_{i}+a s_{i}$ for some $t_{i} \in N$ and $s_{i} \in M$.

Also $N+b M \subseteq(N: a)$. Therefore $(N: a)$ is finitely generated, that is, $(N: a)=\sum_{j=1}^{m} R y_{j}$.

It now follows that $N$ is generated by $\left\{t_{1}, \ldots, t_{n}, a y_{1}, \ldots, a y_{m}\right\}$, which is a contradiction. Hence $I$ is prime. As $N$ is a proper submodule of $M$ and $\operatorname{ann}_{R}(M) \subseteq I$, by [5, Corollary 2.11], $N=I M$ is prime. Which again is a contradiction.

We now recall the following result, which is due to M. Isaacs (see, Ex.2.23 of [4]): Let $R$ be a ring such that every prime ideal of $R$ is principal. Then $R$ is a principal ideal ring.

However, if every prime submodule of an $R$-module $M$ is cyclic then every submodule of $M$ is not necessarily cyclic. For example, in a two dimensional vector space $V$ over a field $k$ every proper subspace is cyclic.

As $V$ is not a multiplication $k$-module in the above case, one naturally wonders if the result is true for multiplication modules. Unfortunately, that is not the case, as can be seen from the following example:

Example 3.3 First note that if $R$ is a Dedekind domain then every ideal of $R$ is a multiplication ideal. If, in addition, the ideal class group of $R$ is of order 2 then the product of any two non-principal ideals is principal.

Now let $A=\mathbb{Z}[\delta]$, where $\delta$ denotes the complex number $\sqrt{-5}$. Then $A$ is a Dedekind domain which is not a principal ideal domain. In fact, the ideal $(2, \delta-1)$ is not principal. Furthermore, the ideal class group of $A$ is of order 2 (for details, see, [1, pp. 414-431]).

Let $S_{0}$ denote the set of all nonzero prime elements of $A$ and let $S$ denote the multiplicative set generated by $S_{0}$. Put $R=S^{-1} A$. Then $R$ is still a Dedekind domain with ideal class group of order 2 . Hence the product of any two non-principal ideals of $R$ is principal. One easily verifies that if $P$ is a nonzero prime ideal of $A$ with $P \cap S=\emptyset$, then $S^{-1} P$ is not principal in $R$. In other words, no nonzero prime ideal of $R$ is principal. Let $I$ be a non-principal ideal of $R$. If $N$ is prime submodule of $I$ then $N=P I$ for some prime ideal $P$ of $R$, and therefore $N$ is cyclic.

In the next result we give some sufficient conditions for every submodule of a multiplication module $M$ to be cyclic, provided its all prime submodules are cyclic. 
Theorem 3.4 Let $R$ be a ring and $M$ be a faithful multiplication $R$-module such that every prime submodule of $M$ is cyclic. Then every submodule of $M$ is cyclic in each of the following cases:

(a) If $R$ is semi-local.

(b) If there exists a non zero-divisor $a \in R$ such that $R a$ is a maximal ideal of $R$.

(c) If every proper submodule of $M$ is cyclic.

(d) If there exists an onto map $\phi: M \longrightarrow R$.

Proof. By Theorem 3.2, $M$ is Noetherian and therefore so is $R$.

In each of the cases it is enough to show that $M$ is cyclic. For, if $M$ is cyclic then $M \cong R$ and hence every prime ideal in $R$ is principal. By M. Isaac's Theorem, every ideal of $R$ is principal and the result follows.

Part (a) follows by [2, Proposition 4].

We now prove (b). Let $\mathfrak{m}=R a$. As $\mathfrak{m}$ is maximal, by [5, Theorem 2.5], $\mathfrak{m} M$ is a maximal submodule of $M$ and hence it is prime. Therefore $\mathfrak{m} M=R x$ for some $x \in M$. Let $y \in M \backslash \mathfrak{m} M$. Then $M=\mathfrak{m} M+R y=R x+R y$. As $\mathfrak{m}=(R x: M)$, we have $a y=b x$ for some $b \in R$.

We claim that $b \notin \mathfrak{m}$. Suppose $b \in \mathfrak{m}$. Then $b=r a$ for some $r \in R$. Now $b x=\operatorname{rax}=a y$, that is, $a(r x-y)=0$. As $a$ is a nonzero divisor of $R$, by [5, Lemma 4.1], $a$ is a nonzero divisor of $M$ and therefore we have $y=r x \in \mathfrak{m} M$, a contradiction.

Hence $R a+R b=R$. Therefore there exist $\alpha, \beta \in R$ such that $\alpha a+\beta b=1$. As $x=a(\alpha x+\beta y)$ and $y=b(\alpha x+\beta y)$, we have $M=R(\alpha x+\beta y)$, that is, $M$ is cyclic.

We prove (c) now. If every nonzero divisor of $R$ is a unit then $R$ is semilocal and hence by part (a), $M$ is cyclic.

Therefore, we may assume that $R$ contains a nonzero divisor $a$, which is not a unit of $R$. As $M$ is a finitely generated faithful $R$-module, $a M \neq M$ and therefore $a M$ is cyclic. By [5, Lemma 4.1], $M \cong a M$, that is, $M$ is cyclic.

We now prove $(\mathrm{d})$. Let $K=\operatorname{ker}(\phi)$. Since $M$ is a multiplication module $K=I M$ for some ideal $I$ of $R$. Then $I=(0)=\phi(K)$, that is, $\phi$ is an isomorphism.

Remark $3.5 \quad$ (1) Note that in a two dimensional vector space every proper subspace is cyclic. Therefore, Theorem 3.4(c) does not hold for arbitrary modules. 
(2) If all proper submodules of a module $M$ are cyclic then $M$ need not even be finitely generated. For example, let $p$ be a positive prime and let $G=\left\{a \in \mathbb{C} \mid o(a)=p^{r}\right.$ for some $\left.r \geq 0\right\}$. Then $G$ is an infinite abelian torsion group in which every proper subgroup is cyclic. Clearly, $G$ can not be finitely generated.

The following result is of some interest in view of Example 3.3.

Theorem 3.6 Let $R$ be an integral domain. If there exists a faithful multiplication $R$-module $M$ such that every prime submodule of $M$ is cyclic then $R$ is a Dedekind domain.

Proof. By Theorem 3.2, $M$ is Noetherian and therefore so is $R$.

We may assume that $R$ is not a field.

Let $P$ be a nonzero prime ideal of $R$. Then $M_{P}$ is a nontrivial multiplication $R_{P}$-module. As $R_{P}$ is local, by [2, Proposition 4], $M_{P}$ is cyclic and hence $R_{P} \cong M_{P}$. Therefore $R_{P}$ is a principal ideal domain, that is, a discrete valuation ring for every prime ideal $P$ of $R$. Hence $R$ is a Dedekind domain.

Acknowledgements. The first and the third authors are CSIR SRF and JRF respectively. Their financial assistance is duly acknowledged.

\section{References}

[1] M. Artin, Algebra, Prentice Hall of India, New Delhi, 1991.

[2] A. Barnard, Multiplication modules, Journal of Algebra, 71 (1981), 174-178.

[3] M. Behboodi and H. Koohy, On minimal prime submodules, Far East J. Math. Sci., 6, No. 1 (2002), 83-88.

[4] D. Eisenbud, Commutative algebra with a view toward algebraic geometry, Springer-Verlag, New York 1994.

[5] Zeinab Abd El-Bast and Patrick F. Smith, Multiplication modules, Communications in Algebra, 16, No. 4 (1998), 755-779.

[6] C. P. Lu, Unions of prime submodules, Houston J. Math., 23, No. 2 (1997), 203-213.

Received: February 16, 2007 